Une mairie dans la France coloniale. Koné, NouvelleCalédonie de Benoît Trépied

\title{
Benoît Carteron
}

\section{OpenEdition}

1 Journals

Édition électronique

URL : http://journals.openedition.org/jso/6744

DOI : $10.4000 /$ jso.6744

ISSN : $1760-7256$

\section{Éditeur}

Société des océanistes

\section{Édition imprimée}

Date de publication : 31 décembre 2012

Pagination : 271-273

ISBN : 978-2-85430-033-8

ISSN : 0300-953x

\section{Référence électronique}

Benoît Carteron, « Une mairie dans la France coloniale. Koné, Nouvelle-Calédonie de Benoît Trépied », Journal de la Société des Océanistes [En ligne], 135 | 2012-2, mis en ligne le 09 décembre 2012, consulté le 24 septembre 2020. URL : http://journals.openedition.org/jso/6744 ; DOI : https://doi.org/10.4000/ jso.6744

\section{(C) Tous droits réservés}


Hagen Bernhard, 1899. Unter den Papua's. Beobachtungen und Studien über Land und Leute, Thier- und Pflanzenwelt, Wiesbaden, Kreidel.

HARDING Thomas G., 1967. Voyagers of the Vitiaz Strait. A Study of a New Guinea Trade System, Seattle and London, University of Washington Press.

Hubers Hubert S.V.D., 1942-1945. Kleine musikethnologische Beiträge von der Insel Karkar in Neuguinea, Anthropos, Band 37/40, Heft 1/3, pp. 122-126.

Kunze Georg s.v.D., 1896. Karkar oder Dampier Insel, Petermanns Geographische Mitteilungen XLII, pp. 193-195.

McSwain Romola, 1994. Kulbob and Manub: Past and Future Deities of Karkar Island, Pacific Studies 17, 4, pp. 11-28.

Ter Keurs Pieter, 2006. La Baie de l'Astrolabe, in Philippe Peltier et Floriane Morin (éds), Ombres de Nouvelle-Guinée. Arts de la grande île d'Océanie dans les collections Barbier-Mueller, Paris, Somogy, pp. 136-149.

Gilles BounOuRE

TRÉPIED Benoît, 2010. Une mairie dans la France coloniale. Koné, Nouvelle-Calédonie, Paris, Karthala, postface de Frederick Cooper, 391 p., bibliogr., cartes, tableaux, ill. photogr. noir et blanc.

À partir d'une enquête ethno-historique menée dans la commune de Koné, l'auteur se donne pour projet d'expliquer le succès de l'Union calédonienne (UC) jusqu'au virage indépendantiste de 1977, parti politique prônant l'union multiraciale tout en demeurant « apparemment aveugle aux rapports de pouvoir hérités de l'ère coloniale » (p. 9). L'ethnographie et la micro-histoire permettent ici de prendre en compte les points de vue kanak et non kanak dans l'articulation de leurs enjeux politiques respectifs et de "saisir concrètement, et dans leurs multiples dimensions, les paradoxes, tensions et solidarités au fondement de l'Union calédonienne, telle qu'elle se donnait à voir dans la commune de Koné des années 1950 à 1970 » (p. 11). L'ouvrage est divisé en quatre parties comprenant chacune trois chapitres. Les deux premières parties sont consacrées à l'entrée des Kanak en politique avec l'accès à la citoyenneté en 1946, les deux autres sont centrées sur l'implication des autres habitants dans l'uC.

Avant 1946, la citoyenneté civique et politique kanak s'exerçait dans les configurations de pouvoir spécifiques, à l'écart de l'activité démocratique transposée par l'État dans la colonie aux niveaux communal et régional. Les structures d'autorité de cette période, autour de positions intermédiaires entre le monde kanak et colonial : " chef » (au service de l'administration), responsable religieux (au service des missionnaires) et gardien de bétail (au service des colons) ont servi de point d'appui à l'entrée des Kanak dans la municipalité dans les années 1950, tout en étant cumulées par les mêmes personnes ou des parents proches.

Pour comprendre la complexité de la structure sociale et du pouvoir kanak derrière les médiateurs politiques kanak apparaissant sur la scène publique durant la période de l'indigénat et après l'accès à la citoyenneté (élus, militants, chefs administratifs et religieux, stockmen, lettrés), Benoît Trépied se penche sur la trajectoire politique des membres de trois familles étroitement liées (Poadja, Goroépata et Gorohouna) des clans Görötû et Nädù. L'ordre colonial instauré en 1869 (obligations de travail et interdictions de déplacements) ne s'est vraiment imposé qu'à l'entre-deuxguerres, à l'issue du soulèvement de 1917 et de la répression qui s'en suivit. Avant comme après 1917, l'histoire de la chefferie Nädù-Görötû est travaillée par les tensions entre solidarité vis-à-vis de l'extérieur et concurrences internes. Dans les années 1920, les leaders kanak Katélia Poadja, Auguste Goroépata et Dubos Gorohouna devinrent les relais des missions protestantes et catholiques, renforçant leur position d'intermédiaire et leur proximité plus ancienne avec le monde colonial comme stockmen. Les membres de l'ensemble Nädù-Görötû pérennisèrent ainsi leurs positions sociales et politiques dominantes.

Après la Seconde Guerre mondiale, les nouveaux leaders issus de ces familles participèrent à la mise en place de l'Union calédonienne, en relation étroite avec les responsables religieux, acteurs incontournables de la première décennie de citoyenneté politique mélanésienne. L'auteur revient sur l'influence du Parti communiste calédonien à l'échelle locale dans l'immédiat après-guerre, contrecarrée au profit des associations catholique et protestante anticommunistes (UICALO et AICLF), contribuant tout au long des années 1950 à canaliser le vote kanak en faveur de l'Union calédonienne. La pérennité de l'influence de l'ensemble Nädù-Görötû se maintint ainsi dans l'espace politique municipal, malgré les clivages suscités par l'épisode communiste et la crise interne à l'UC (scission protestante) de la fin des années 50 qui, chaque fois, ont réactivé les anciennes rivalités entre clans et entre chefferies. Comme lors de la nouvelle crise de l' UC en 1977 (motion en faveur de l'indépendance) et lors des affrontements des années 1980, les oppositions partisanes internes se sont mêlées aux héritages familiaux sans les déterminer complètement. Ainsi, « la rencontre entre un contexte historique [...] et des dispositions politiques transmises de génération en génération contribuait à faire des membres des clans Nädù et Görötû tout à la fois des héritiers et des acteurs, à l'intérieur d'un champ des possibles renouvelé en chacune de ces occasions historiques » (p. 194).

Un des premiers effets de l'entrée de l' UC dans la municipalité de Koné à partir de 1954 (cinq élus kanak et deux élus européens sur quatorze conseillers, puis douze élus en 1961) a été la construction de conduites d'eau potable et de routes dans les tribus, venant compenser l'inégalité de traitement due à la gestion séparée des tribus et des villages sous l'ordre colonial. Les adductions d'eau en tribu ont constitué un changement d'envergure pour les populations et « un effet 
palpable et concret de l'octroi de la citoyenneté aux Kanak » (p. 76).

L'entrée dans le jeu politique et la représentation élective a supposé la médiation entre deux structures de pouvoir et la négociation de rapports nouveaux entre la chefferie et les représentants kanak au conseil municipal. Tandis que les premiers élus municipaux étaient également des chefs administratifs de leur tribu, les élus kanak des années 1960 ont mis à profit leur position intermédiaire (temps passé au contact des Européens, en retrait des chefs et responsables religieux) et des ressources nouvelles (linguistiques et financière notamment) dans l'exercice des responsabilités municipales. Dans la logique des changements alors en cours : travail salarié, urbanisation, mobilité des habitants, la politique municipale s'est progressivement autonomisée vis à vis des autorités tribales ; la légitimité professionnelle de salariés et scolaire d'instituteurs se substituant à la légitimité coutumière et religieuse jusqu'à l'émergence des premiers intellectuels ayant fait des études en France dans les années 1970.

Le système social à l'échelle communale a été structuré par les tensions économiques (rapports de classe) et les tensions raciales (relations Blancs / Kanak / Asiatiques recoupées par les relations colons / indigènes / travailleurs engagés venant d'autres colonies). Après la Seconde Guerre mondiale, l'Union calédonienne s'est positionnée au cœur de ces clivages, comme protectrice des petits contre les gros et comme tentative de dépassement des hiérarchies coloniales et raciales.

L'auteur restitue d'abord l'émergence du monde colonial et ses clivages internes dans la région de Koné, des premières installations (1875) jusqu'à la fin du régime de l'indigénat et de l'engagement. Dépourvus de moyens, soumis aux aléas, les "petits colons» étaient dépendants de l'administration et d'une minorité de gros exploitants (éleveurs) ou alors se diversifiaient avec des activités complémentaires (artisanat, emplois salariés : mine, petite fonction publique). Le maintien des petits colons a été en outre permis par l'existence d'une main d'œuvre soumise et exploitée : condamnés et libérés du bagne, indigènes et travailleurs sous contrats (Javanais, Tonkinois). Le tableau de la population de Koné avant 1946 est celui de cette stratification sociale poussée, mêlant de façon nuancée richesse économique, statut juridique, race et nationalité (expatriés japonais et Européens issus du bagne étaient au-dessus des engagés et Kanak par exemple).

Élu tout d'abord député en 1951 grâce aux seules voix des Kanak, le créateur-leader de l'uc, Maurice Lenormand, élargit son audience au vote européen en prenant la défense des « petits » contre les « gros », des commerçants (maisons Ballande et Magnier notamment) qui trustaient la représentation politique à l'échelle locale et du territoire colonial. Le soutien électoral de ces derniers était assuré dans la période 1880-1950 par l'endettement chronique des producteurs et la dépendance aux circuits commerciaux. La stratégie, qui consistait non pas tant à rejeter les clientélismes qu'à capter des réseaux de clientèles de marchands plus modestes, ne réussit pas à l'échelle de Koné où les producteurs agricoles, malgré leur dépendance, restèrent fidèles aux grandes maisons commerciales jusqu'aux années 1970.

La mobilisation par l'UC des ouvriers, moins concernés par les logiques clientélistes des grandes maisons commerciales, s'est réalisée par le biais de l'action syndicale. Maurice Lenormand s'appuya avec succès sur l'opposition des mouvements syndicaux au patronat industriel. Après la Seconde Guerre mondiale, la condition ouvrière s'est améliorée tandis que la mine de chrome de Thiébaghi, $100 \mathrm{~km}$ au nord de Koné, devenait le nouveau pôle économique attractif où une sociabilité ouvrière "multiraciale » s'est développée. L'action syndicale contribua à l'émergence d'une conscience de classe, notamment avec les grandes grèves de 1955-56. Cas à part parmi les colonies françaises, le syndicalisme ouvrier était principalement mené par des leaders européens. C'est ainsi en s'appuyant sur les syndiqués européens et asiatiques que l'uc a élargi son assise électorale, créant un rapprochement politique inédit entre autochtones et autres habitants par la prise en charge conjointe de la « question indigène » (dans une perspective modérée et réformiste) et de la défense des « petits » (avec un discours plus à gauche).

Cette double composante marquait en même temps la limite du rapprochement opéré par Maurice Lenormand et l'UC: on pouvait s'accommoder d'une vision modérée du traitement de la question autochtone, inspirée des missions religieuses, sans remettre fondamentalement en cause l'héritage colonial. La réussite électorale de l'uc reposait en définitive sur une séparation tacite : aux Européens la lutte sociale d'inspiration syndicale, aux associations missionnaires la question de la défense de la place des Kanak à laquelle les Européens restaient relativement indifférents ; une alliance à moindre coût dont l'auteur tire la conclusion que « le slogan "deux couleurs, un seul peuple" ne semblait représenter concrètement qu'un argument électoral, sans fondement sociologique ni réel projet politique » (p. 278).

Dans la dernière partie de l'ouvrage, Benoît Trépied met à l'épreuve ce constat du décalage entre la devise du parti et la réalité sociale et politique par l'examen de la trajectoire d'élus municipaux et militants européens de l'uc à Koné. Il s'agit d'appréhender le poids des hiérarchies coloniales et raciales chez ces militants, alors que ce sont les anciens «stigmatisés de l'époque coloniale » (p. 284) qui ont pris le contrôle du conseil municipal dans la seconde moitié $\mathrm{du} \mathrm{Xx}^{\mathrm{e}}$ siècle.

L'étude des rapports entre Blancs de l'uc et Kanak soulève la question épineuse du racisme anti-Kanak d'avant les affrontements violents des années 1980. L'auteur souligne d'abord la difficulté à appréhender une telle question prise à partir d'entretiens rétrospectifs alors qu'elle était toujours reliée à des enjeux politiques. Le recoupement des sources sur les cas d'élus blancs de l'uc à Koné laisse cependant entendre leur participation au racisme ambiant dans un contexte d'allant de soi de la domination coloniale, mais tout en entretenant par ailleurs des relations de proximité (métissages, parents intégrés au monde 
kanak, activités communes, familiarité, capacité à se réconcilier après les conflits) et en menant une action favorable aux Kanak. L'auteur en conclut à « l'ambiguïté fondamentale des relations entre Blancs et Noirs dans la région de Koné, à la fois structurellement inégalitaires et pourtant composées de multiples interactions inter-individuelles qui pouvaient, dans certaines situations bien particulières, déborder du cadre colonial général » (p. 314). Tout en reconnaissant les limites d'une étude qui nécessiterait une approche très fine, l'auteur souligne la nécessaire distinction entre un racisme cantonné à des représentations négatives (paternalisme, vision approximative du monde kanak, indifféren-

ciation des individus) qu'on retrouve chez les militants blancs de l'Uc, et des violences physiques qui étaient toujours le fait d'individus refusant toute alliance politique avec les Kanak.

Le dernier chapitre examine le parcours de deux hommes de statut civil commun qui ont incarné l'uc à Koné de par leur leadership : Lucien Allard et William Yoshida. Lucien Allard, maire de la commune de 1961 à 1970, a d'abord noué des relations professionnelles inédites avec les responsables kanak, sur le mode de la collaboration et non plus de la contrainte, en s'installant à Koné aussitôt la Seconde Guerre mondiale comme chef des travaux publics. William Yoshida, premier adjoint de 1970 à 1980, métis né d'un père japonais et d'une femme kanak, marié avec une Javanaise, commerçant réputé à la mine de Tiébaghi puis dans le village de Koné, était à même de franchir les frontières raciales par son insertion conjointe dans des univers sociaux habituellement étanches les uns aux autres. Il a pu ainsi remplir un rôle de médiateur permettant d'activer l'alliance entre militants kanak, européens et asiatiques de l'UC. Les liens privilégiés établis par ces leaders avec les habitants kanak des tribus ont constitué l'assise de l'alliance politique propre au parti.

Cependant, la mise en avant sur la scène publique de ces leaders, incarnation du slogan multiracial du parti, masquait l'écart entre les projets politiques, les contradictions coloniales, ainsi que les ségrégations raciales des militants de base. L'auteur s'en réfère à Isabelle Merle (1995) et Adrian Muckle (2004) pour rappeler une véritable unité européenne qui s'est construite dans l'histoire de la colonisation par opposition à la « sauvagerie » indigène et par la crainte, régulièrement réactivée, de la révolte kanak. La prégnance des anciennes frontières coloniales et raciales a ainsi dépassé les clivages entre pro et anti-uc au sein du monde blanc. De plus, après 1946, les oppositions libres/condamnés, citoyens/nationaux non citoyens, ont été substituées par la seule opposition entre citoyens de statut civil (descendants de colons, ex-sujets français et étrangers naturalisés) et citoyens de statut coutumier réservé aux Kanak, jusqu'à devenir le seul clivage persistant par-delà les années 1970.

Benoît CARTERon, Université catholique de l'Ouest, Angers
Hamson Michael, 2011. Art of the Boiken, contributions by Paul Roscoe, Mike Glad, Ron May, Helen Dennett and Patricia May, Palos Verdes, M. Hamson, 264 p., bibliogr., cartes, 178 objets reproduits, nombreuses autres ill.

Quoi qu'il faille penser des « initiatives privées » et commerciales dans un domaine de connaissance par excellence public, on ne saurait contester que la nouvelle publication de M. Hamson (voir Jso 129 et 133 pour ses deux précédents volumes) contribue à éclairer une des nombreuses «zones grises » qu'offre encore l'histoire des arts des environs du fleuve Sepik (Papouasie Nouvelle-Guinée) malgré tous ses progrès récents. Il serait malaisé de disputer (pour ne pas dire « marchander ») à ce marchand d'art, à l'occasion d'un livre-catalogue dont près de la moitié des pièces reproduites sont exclues des circuits commerciaux, le mérite d'avoir réuni et porté à la connaissance de qui le souhaite les travaux d'excellents spécialistes sur une région culturelle restée trop peu étudiée par la recherche officielle. Il est même possible, et souhaitable, que cette dernière en tire quelque profit.

L'éditeur a toute raison de relever, dans son introduction (pp. 7-8), les attributions confuses réservées jusqu'à ces dernières années aux objets issus de cette région définie par sa langue (de la famille $\mathrm{Ndu}$ ), souvent décrits comme «Yangoru» (un de ses sept groupes dialectaux), "Arapesh » (voisins du nordouest), "Abelam » (voisins du sud-ouest) ou même encore plus vaguement «Bas-Sepik» (à quelques dizaines de kilomètres au sud et à l'est). Néanmoins l'indication de provenance Boiken est devenue un peu plus fréquente dans les publications académiques ou commerciales, notamment grâce aux travaux de P. B. Roscoe, dont le premier séjour sur ce terrain remonte aux années 1979-1981.

Tel est aussi le grand spécialiste auquel M. Hamson a demandé un résumé de la culture et des arts boiken («The Boiken Culture», pp. 10-13, «The Affecting Presence of Boiken Art », pp. 40-48), dont on appréciera la concision et le refus motivé des interprétations symboliques ou métaphysiques de leur répertoire iconographique. Sans développer tout ce qu'il a pu établir de la préhistoire des Boiken ou des mœurs de leurs voisins Arapesh, plus guerrières que ne les décrivait Margaret Mead (voir notamment ses articles de 1989 et 2003, non mentionnés dans l'ouvrage), il présente cette société comme principalement éprise de démonstrations de force, visant à inspirer aux autres des sentiments mêlés de trouble, d'humiliation et de jalousie, et à renforcer ses propres représentations de puissance menaçante.

Entre ces deux contributions de P. B. Roscoe s'intercale un «portfolio » (pp. 14-39) d'un photographe renommé, M. Glad, qui a parcouru l'aire boiken avec l'éditeur en mai 2010 pour observer la vie quotidienne aussi bien sur les côtes (Boiken, Wewak) et les îles (Mushu) que dans l'arrière-pays (Passam, Kubalia, Yangoru et environs). On peut regretter de ne pas 